\title{
Da bleibt einem doch die Spucke weg!
}

Die Ursache von Halitosis (Mundgeruch) ist bei jedem 4. Erwachsenen Xerostomie, Mundtrockenheit. Neben Mundgeruch führt sie auch zu einer verminderten Remineralisation der Zahnhartsubstanz. Die gute Nachricht: „Häusliche ProphylaxeMaßnahmen wie der Einsatz bestimmter ätherischer Öle wie Eucalyptol, Thymol und Krauseminzöl regen nachweislich die Speichelproduktion an und lindern so effektiv Mundtrockenheit und können so unterstützen, um Begleiterscheinungen von Xerostomie zu verringern“, so Dr. Frank Marahrens, Zahnarzt aus Leinfelden-Echterdingen. Eine Zahncreme, die diese Öle enthält, wie etwa das medizinische Zahncreme-Konzentrat Ajona, sorgt zusätzlich für langanhaltend frischen Atem. Die verwendete Zahncreme sollte zudem antibakteriell wirken und ebenso effektiv wie schonend reinigen. Beide Kriterien erfüllt das medizinische Zahncreme-Konzentrat. „Mikrobiell betrachtet sind hauptauslösend für Mundgeruch die Gruppe der Lactobazillen, der Streptokok- ken und auch Pilze wie C. albicans“, so Marahrens. Gegen diese Bakterienstämme und auch Pilze wirkt Ajona nachweislich antimikrobiell. Der RDA-Wert, der über die Abriebwirkung einer Zahncreme Aussage trifft, liegt bei Ajona bei niedrigen 30 . Das Zahncreme-Konzentrat begegnet damit der krankheitsbedingt verminderten Remineralisierung des Zahnschmelzes auf sanfte Weise. Karies und Halitosis infolge von Xerostomie können effektiv vermieden werden. Übrigens: reine Kamillenblüten, in Deutschland gepflückt, sind eines der "Geheimnisse“ von Aminomed. Die medizinische Kamillenblüten-Zahncreme beugt Zahnfleischentzündungen natürlich medizinisch vor. Auf dem neuen Aminomed-One-Pager - für mobile Endgeräte und Tablets optimiert - präsentiert sie sich erstmals auf einer eigenen Site umfassend im Netz. Hersteller Dr. Liebe hat alle wichtige Fachinfos kompakt zusammengestellt und reich illustriert. Sie benötigen Zahnpflegetipps für Ihre Patienten? Dann können Sie ganz komfortabel das entsprechende PDF von dem One-Pager herunterladen. Klicken Sie doch einfach mal rein: http://www.aminomed.de

Nach einer Pressemitteilung der Dr. Rudolf Liebe Nachf. GmbH \& Co. KG, Leinfelden-Echterdingen 